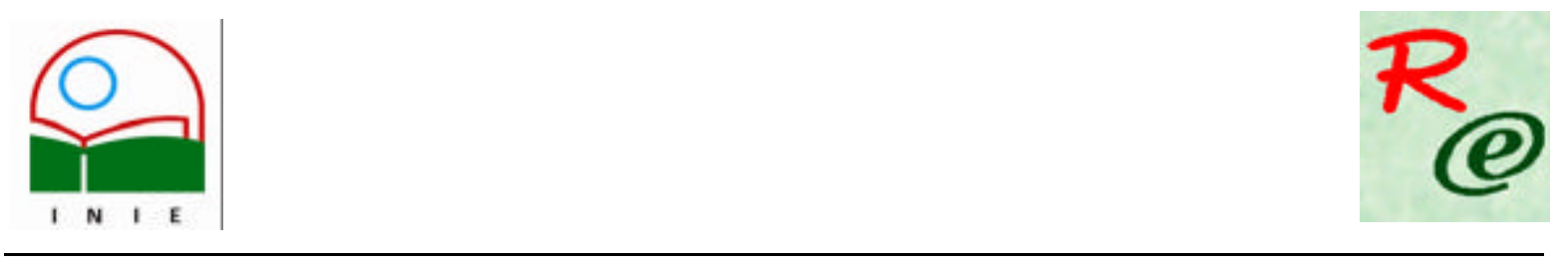

Actualidades Investigativas en Educación

Revista Electrónica publicada por el

Instituto de Investigación en Educación

Universidad de Costa Rica

ISSN 1409-4703

http://revista.inie.ucr.ac.cr

COSTA RICA

\title{
REDES COMUNITARIAS: UN DESAFÍO PARA ESCUELAS
} URBANO-MARGINALES

COMMUNITY NETWORKS: A CHALLENGE FOR URBAN MARGINAL SCHOOLS

Volumen 9, Número 1

pp. $1-33$

Este número se publicó el 30 de abril 2009

Mirta Giacobbe Tovani

Nora Moscoloni Bicchi

La revista está indexada en los directorios:

LATINDEX, REDALYC, IRESIE, CLASE, DIALNET, DOAJ, E-REVIST@S,

La revista está incluida en los sitios:

REDIE, RINACE, OEI, MAESTROTECA, PREAL, HUASCARAN, CLASCO 


\title{
REDES COMUNITARIAS: UN DESAFÍO PARA ESCUELAS URBANO-MARGINALES COMMUNITY NETWORKS: A CHALLENGE FOR URBAN MARGINAL SCHOOLS
}

\author{
Mirta Giacobbe Tovani ${ }^{1}$ \\ Nora Moscoloni Bicchi
}

\begin{abstract}
Resumen: Las ideas básicas de este trabajo son conocer y comprender el entramado de la red de relaciones entre los distintos actores de las instituciones: escuela y organismos de la comunidad y su incidencia en la calidad educativa / calidad de vida. Analizamos la visión de los distintos actores educativos acerca de la inserción de la escuela en la comunidad. Tomamos como punto de partida el análisis de las relaciones entre calidad educativa y calidad de vida, en poblaciones urbano-marginales. Realizamos en primer lugar una investigación exploratoria, para conocer las características generales de escuelas que reciben población marginal. En una segunda etapa seleccionamos cuatro escuelas, que tomamos como casos e indagamos sus problemáticas. En ellas observamos gran dificultad para la construcción de redes comunitarias desde la institución escolar. Si bien responden a los problemas puntuales, mediante cambios o innovaciones planificados dinámica y flexiblemente, no existen soluciones duraderas debido a la falta de una convocatoria que comprometa a todas las instituciones de la comunidad constituyendo una efectiva relación en red. Estos resultados alcanzados en la investigación son producto de la triangulación de los datos estadísticos, documentales e históricos así como de los procedentes de la observación participante y de la información de las entrevistas en profundidad.
\end{abstract}

\section{Palabras claves: COMUNIDAD, RED COMUNITARIA, EDUCACIÓN DE CALIDAD, CALIDAD DE VIDA}

\begin{abstract}
The basic ideas of this work are to know and understand the fabric of the network of relationships between the different institutions involved in school and community agencies and their impact on the quality of education / quality of life. The vision of the different actors of education about the insertion of the school in the community is analyzed from the point of view of the school. The analysis started studying how the quality of education is related to the quality of life in marginalized urban populations. At first an exploratory research was made to learn about the general characteristics of schools that receive marginal population, and then four schools were selected from all, to study them as cases looking for their problematic nodes. Although they respond to precise problems by means of changes or planned innovations dynamically and flexible, do not exist lasting solutions due to the lack of a call to institutions of the community in order to get involved so as to constitute an effective relation in network. These results achieved come from the triangulation of statistical, documental and historical data as well as from participant observation and information from in depth interviews
\end{abstract}

Key words: COMMUNITY, COMMUNITY NETWORK, EDUCATION OF QUALITY, QUALITY OF LIFE

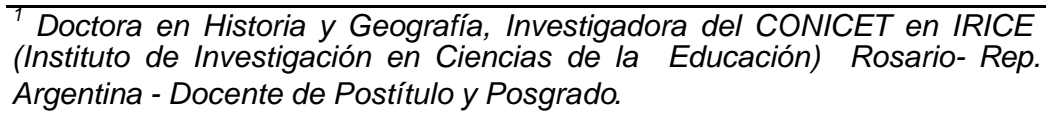

Dirección electrónica: giacobbe@ciudad.com.ar (part.) - giacobbe@iriceconicet.gov.ar (laboral)

${ }^{2}$ Doctora y docente investigadora de la Facultad de Ciencia Política de la Universidad Nacional de Rosario, Estadística especializada en análisis multidimensional de datos en IRICE (CONICET-UNR), docente en Maestrías y Doctorados.

Dirección electrónica: moscoloni@irice-conicet.gov.ar

Artículo recibido: 31 de octubre, 2008

Aprobado: 27 de abril, 2009

Volumen 9, Número 1, Año 2009, ISSN 1409-4703 


\section{Introducción}

En los sectores urbano-marginales, la escuela sigue siendo una de las pocas instituciones que sirve de referente válido y reconocido. Por ello nuestra meta es instaurar la institución educativa -gestora y consolidadora de conocimientos- en la comunidad, con el comienzo de acciones para una formación/ transformación cooperativa sostenible en la calidad de vida.

Procuramos, por un lado, conocer la internalización de la responsabilidad que tiene cada uno de los actores de ejercer el protagonismo correspondiente a su rol, no como individuo aislado, sino interactuando en el seno de una comunidad, que se conforma por ellos y al mismo tiempo los conforma. Por el otro, fortalecer la red de relaciones entre los actores sociales de la escuela y entre ésta y las instituciones de la comunidad, atendiendo a las problemáticas socio-educativas locales y regionales, en la búsqueda de una educación de calidad para una mejor calidad de vida.

\subsection{Objetivos de investigación}

- Desarrollar un proyecto de investigación a partir del eje escuela-comunidad, apostando a la calidad de vida en una sociedad en crisis.

- Conocer la institución educativa y sus interrelaciones contextuales con la comunidad, en los asentamientos irregulares de los barrios de la ciudad de Rosario

- Conocer el entramado de la red de relaciones intra e interinstitucional entre los distintos actores y la incidencia en la calidad educativa/ calidad de vida

\section{Referente Teórico}

Desde diferentes teorías se enfatiza acerca del lugar clave que ocupa la institución escolar respecto de las políticas curriculares de mejora. Este posicionamiento implica una nueva perspectiva: la prioridad es producir cambios a nivel de organización de las instituciones educativas, para que aquellas transformaciones curriculares de mejora de los aprendizajes se sostengan y adquieran un desarrollo estable y sostenible.

Se trata de fortalecer la capacidad interna de cambio de cada escuela para que pueda desarrollar una cultura innovadora, por esta razón se justifica un trabajo permanente sobre las fortalezas para sustentar las novedades. Los cambios en la familia y en las pautas de socialización se tradujeron en permanentes desafíos para la escuela que recibe niños cada vez más diferenciados en sus formas de actuar y de pensar, "con distinto capital cultural". 
Esto, sumado a la transformación de la familia y la extensión de los medios de comunicación, generaron una profunda transformación en las formas de acceso de los niños al tipo de información.

Entre los aportes teóricos conceptuales y estratégicos del nuevo planteo del llamado movimiento de mejora en las prácticas escolares más relevantes, se destaca la convicción de que es posible que la escuela pueda marcar una diferencia sustantiva en la historia escolar de los aprendizajes de los alumnos en su propia dinámica.

Las investigaciones actuales demuestran que la estructura de la escuela, su clima general académico y de trabajo, la gestión de las direcciones, las expectativas positivas o no de docentes sobre el aprendizaje de la población estudiantil y los tipos de estrategias de aula de los docentes, tienen la capacidad de provocar diferencias fundamentales en los aprendizajes de los alumnos y alumnas. A la vez, los políticos y los profesionales consideran que los cambios educativos tienen que ver con el rol de los equipos directivos y de supervisión, conjuntamente con la profesionalización docente en la búsqueda de recuperar la identidad y la capacidad de formación de la escuela. El contexto, en estos tiempos, no deja aséptica a la escuela, penetra en la misma, a través de valores, creencias, normas, mitos, rituales, costumbres, estereotipos, eslóganes, etc. Lo social penetra por ósmosis en las prácticas cotidianas, es difícil sustraerse a su influencia, por eso la escuela debe abrirse a la comunidad.

Cada escuela, por otra parte, necesita solucionar problemas propios, porque cada una es una identidad organizativa. Los cambios o innovaciones surgen como respuesta exclusivamente a esos problemas, para lo cual se necesita una alta cuota de creatividad y distinción en un proceso continuo. Para planificar un cambio es necesario antes detectar los problemas institucionales, así como las causas que los originan y concretar pasos operativos y viables en el camino hacia la solución.

El cambio se planifica dinámica y flexiblemente -por parte de toda la comunidad educativa- pero contextualizado en un espacio y en un tiempo específico; en el camino se redefinen sus metas y sus estrategias permanentemente. La flexibilidad le permite dar respuesta a las circunstancias cambiantes en un proceso de creación y ajuste permanente.

Actualmente, el concepto de calidad educativa está referido a qué es lo que se aprende en el sistema y a su relevancia en términos individuales y sociales y no tanto a los resultados de aprendizajes efectivamente alcanzados por la acción educativa. En este sentido una educación de calidad es aquélla cuyos contenidos responden adecuadamente a lo que el Volumen 9, Número 1, Año 2009, ISSN 1409-4703 
individuo necesita para desarrollarse como persona y para desarrollarse en los diferentes ámbitos de la sociedad.

Esta dimensión se refiere a la calidad de los procesos y medios que el sistema brinda a los alumnos para el desarrollo de su experiencia educativa. Desde esta perspectiva una educación de calidad es "aquélla que ofrece a los niños un adecuado contexto físico para el aprendizaje, un cuerpo docente preparado para la tarea de enseñar, buenos materiales de estudio, estrategias didácticas adecuadas, etc." (Toranzos, 2000, p. 38)

Los indicadores tradicionales para evaluar el desempeño del sistema educativo aplicando este criterio de calidad- son insuficientes. La preocupación no es exclusivamente la evolución de la matrícula, el nivel de repetición, de deserción escolar, etc, sino qué, quiénes y en qué condiciones se aprende en las escuelas. Es el Ministerio de Educación Nacional quien tiene que diseñar políticas que definan los conocimientos y capacidades cognitivas básicas a alcanzar por todo alumno, para evitar el desmembramiento del sistema. Sin embargo, es necesario que la institución educativa se responsabilice de su propio funcionamiento y de los resultados que obtiene, sin supeditarse a la 'notificación a las instancias superiores'. Lo cual no sólo depende de una modificación de la normativa vigente, sino de cambios de actitud, de posicionamiento y también de capacitación, al respecto.

Una verdadera evaluación, en la dirección de valoración del significado de las acciones y de búsqueda de una interpretación de los sentidos que asuman para los actores, no se reducirá sólo a un sistema de control y podrá tener en cuenta los referentes verdaderamente significativos de la realidad que se intenta valorar.

La evaluación se considera entonces, como una calidad a construir a partir de cada realidad. Desde esta concepción de evaluación, la valoración de la realidad educativa no constituye el fin de la acción de evaluar, sino sólo el punto de partida para favorecer el desarrollo de nuevos proyectos. En los últimos años, algunos autores coinciden en que calidad es un término polisémico, multivariable. Por ejemplo, es definida como: "aquélla (educación de calidad) que responda de manera eficaz a las demandas sociales" (Lugo, 2002, p. 43)

Es una realidad que en las escuelas no se produjo a través del tiempo la modificación de su estructura de organización y de sus procesos de gestión, de acuerdo con los cambios contextuales. La propuesta consiste en apartarnos del paradigma clásico de la escuela para imaginar nuevos escenarios por fuera del ya existente. De lo contrario, corremos el riesgo de 
cambiar para que nada cambie. La propuesta de innovación incorpora el contexto y llega a la práctica institucional. Implica un cambio integral de la escuela.

El término integral alude al concepto de escuela en el que se funda, según el cual el ámbito escolar constituye un entramado abierto y fundamental no sólo para el logro de aprendizajes sino para la promoción social y cultural de los sectores menos favorecidos. La escuela es definida entonces como institución comprometida con el derecho a educarse, y, por ello, con la generación de las condiciones que hagan factible una distribución equitativa de bienes simbólicos (culturales, sociales, pedagógicos) y el fortalecimiento de las condiciones materiales, definidas en diálogo con la comunidad que constituye su contexto. Desde nuestro punto de vista, el concepto de integralidad no puede interpretarse sin una concepción acerca de igualdad, establecida como principio y como punto de partida, tanto de la política educativa como de las prácticas pedagógicas.

Actualmente se reconoce que la educación es un derecho universal, que constituye un elemento fundamental de integración social para toda persona. Se reconoce también que todo niño y niña tiene capacidades y potencialidades singulares, por lo que distintas necesidades exigen respuestas diversas. La educación es parte de y no algo separado de la vida de la niñez. Toda persona en la infancia tiene el derecho a pertenecer a una comunidad y compartir con sus miembros las distintas experiencias de la vida cotidiana; tiene el derecho de acceder a una educación de calidad junto con los otros niños y niñas de su propio barrio; seguramente, tiene una contribución para hacer a la sociedad. Por eso actualmente se habla de educación inclusiva. La cual significa: ..."hacer efectivos para todos los niños, jóvenes y adultos los derechos a la educación, la participación y la igualdad de oportunidades, prestando especial atención a aquéllos que viven en situación de vulnerabilidad o sufren cualquier tipo de discriminación" (UNESCO, 2002)

El principio de educación inclusiva fue adoptado en la Conferencia Mundial sobre educación de necesidades especiales: acceso y calidad (Salamanca, 1994): "todas las escuelas deben acoger a todos los niños independientemente de sus condiciones personales, culturales o sociales; niños discapacitados y bien dotados, niños de la calle, de minorías étnicas, lingüísticas o culturales, de zonas desfavorecidas o marginales". Para ello, es preciso que las escuelas modifiquen sustancialmente su estructura, funcionamiento y propuesta pedagógica con el fin de dar respuesta a la diversidad de necesidades educativas de todos y cada uno de los alumnos. 
La inclusión es más que poner al alumno con alguna necesidad especial en una clase regular, implica minimizar las barreras que impiden la participación y el aprendizaje (UNESCO, 2002)

Una política institucional inclusiva es la que presta atención a la población infantil en situaciones de vulnerabilidad social. Así como la escuela necesita desarrollar una política de inclusión en el aula, de estudiantes con necesidades especiales; también es una realidad que el sistema educativo sigue expulsando sus alumnos y alumnas y que muchos especialmente en zonas vulnerables- se encuentran fuera del sistema. Ante ello, es necesario que la escuela desarrolle sus propios programas de inclusión, articulados con otras entidades del barrio, para atraer nuevamente a su población.

La vulnerabilidad social, es entendida así como:

... una condición social de riesgo, de dificultad, que inhabilita e invalida, de manera inmediata o en el futuro, a los grupos afectados, en la satisfacción de su bienestar -en tanto subsistencia y calidad de vida- en contextos sociohistóricos y culturalmente determinados. (Perona, 2007, p. 5)

La situación de carencia y deterioro no sólo compromete el presente, con el debilitamiento de la trama social sino que involucra a las generaciones futuras, en la perspectiva de la transferencia intergeneracional de la pobreza. Es casi un círculo perverso donde se reproducen las condiciones de marginalidad. Cuando se apela al concepto de carencia para describir una situación de pobreza también se está haciendo referencia al deterioro de los vínculos relacionales que se traduce en un alejamiento de la vida pública donde la presencia política o su influencia social se mantienen en el plano de lo formal antes que en el real. La línea, que entiende pobreza como carencia, es a la que se vinculan las nociones de vulnerabilidad y de exclusión y la posibilidad de pensar si pueden ser herramientas analíticas que permitan una aproximación más dinámica tanto a la diversidad de situaciones a las que se enfrentan los que de una u otra manera son partícipes de algún tipo de privación como para indagar en el proceso por el cual amplios sectores de la sociedad perdieron la participación en una o varias formas de relación social.

Partiendo de esta idea de vulnerabilidad, hablamos de la incapacidad de resistencia cuando se presenta un fenómeno amenazante o la incapacidad de recuperación cuando se transita una crisis profunda. Ella produce inestabilidad, desorientación, inseguridad, incertidumbre, las que exigen variadas estrategias de superación. Debido a la crítica Volumen 9, Número 1, Año 2009, ISSN 1409-4703 
situación económica y social por la que ha atravesado la Argentina, las escuelas se han visto enfrentadas a una diversidad de demandas y problemas que en algunos casos excedieron a sus funciones y posibilidades y que ocuparon el territorio de lo pedagógico con innumerables actividades que las urgencias impusieron. El desafío de superar las debilidades socioeconómicas y su incidencia en el ámbito educativo exigen, además del trabajo de las escuelas y del Estado, el accionar de las familias y de las organizaciones de la comunidad.

La deserción escolar está condicionada por la situación social de los niños y las niñas. Allí es donde la desigualdad se presenta, donde las circunstancias marcan las diferencias y donde las escuelas se ven obligadas a realizar los máximos esfuerzos para cumplir con la tarea de educar. Así, se expresa que:

Las informaciones disponibles parecen sugerir una relación directa y negativa entre las violencias y el desempeño escolar. Se puede suponer que el desempeño de las escuelas -cuyas relaciones se ven perjudicadas por la alta proporción de alumnos que rechazan a sus compañeros - muchas veces es el factor más afectado. (Abramovay, 2005, p. 23)

La violencia influye, también, en los porcentajes de ausentismo y deserción de estudiantes; lo cual lleva a enfrentamientos con otros actores educativos y con los padres y madres. Frecuentemente las personas responsables de hacer cumplir la disciplina escolar no tienen credibilidad ante la población estudiantil, debido a que pasan por alto determinadas acciones. Características puntuales de la escuela tradicional contribuyen a que estas actitudes agresivas y violentas persistan, porque se tiende a minimizar la gravedad de las agresiones, haciendo "como si no existieran", "no pasa nada". Tampoco podemos pensar que: "La violencia en los centros educativos es sólo un síntoma de los graves problemas que afronta nuestra sociedad. No hay respuestas fáciles"(Torres Castro, 2005, p.86).

Los brotes de violencia o de agresividad, en los centros educativos, constituyen un fenómeno al que lamentablemente nos empezamos a acostumbrar y al que cabe interpretar como un reflejo de lo que ocurre en la sociedad. La formulación del concepto de violencia escolar fue incluido en el escenario nacional e internacional como uno de los grandes desafíos a los que hay que enfrentarse actualmente. El término está rodeado de dificultades, porque incluye situaciones muy diversas, desde pequeñas infracciones y ataques a bienes materiales, hasta situaciones que representan riesgos de vida. Pese a esta dificultad conceptual existe un punto de consenso básico, que es que cualquier agresión -física, Volumen 9, Número 1, Año 2009, ISSN 1409-4703 
psíquica o institucional- dirigida contra personas o estructuras mobiliarias o inmobiliarias es considerado un acto de violencia. Partiendo de este consenso se define violencia escolar como:

Un fenómeno heterogéneo que rompe la estructura de las representaciones básicas del valor social: la de la infancia y la de la escuela, así como la de la propia sociedad... las violencias en el universo escolar deben ser jerarquizadas, de forma que sean comprendidas y explicadas. Tal jerarquía se basa en la naturaleza de los actos en cuestión. Hay actos asociados a lo que es llamado violencia (robo, violencia sexual, daños físicos) y hay actos de violencia institucional y simbólica (Abramovay, 2005, p.16)

Es decir, existen distintos grados de violencia, por lo que se propone usar los términos "agresión, comportamiento agresivo, intimidación" para aquellos actos que tienen una connotación emocional y son comunes en la vida de la escuela, distinguiéndolos de los hechos de violencia propiamente dicha. Dichas acciones exhibidas por la niñez y por adolescentes muestran lo que ocurre en su entorno y cuyas causas hay que buscarlas en varios factores entrelazados: sociales, ambientales, relacionales, escolares, familiares y personales. Entre los factores externos, diferentes autores, mencionan los socioeconómicos, exclusión social, colapso de la estructura familiar, maltrato intrafamiliar, tráfico de drogas, etc. Entre los internos, figuran los sistemas de normas y de reglamentos, proyectos políticospedagógicos, baja calidad de la enseñanza, falta de recursos, tensiones vivenciadas en la rutina cotidiana de la escuela. Aunque como antes mencionamos, deben analizarse no aislados, sino entrelazados.

Las conductas agresivas, dentro de la escuela, no se reducen a acontecimientos de violencia física, sino que generalmente se trata de abusos de poder -"modelo dominio/sumisión", pueden ser verbales (violencia a través del lenguaje o "violencia visible a los oídos"), gestuales, de exclusión de actividades comunes, etc.- por parte del más fuerte, sea estudiante, docente, director o directora, etc.

La escuela mediante un trabajo pedagógico comprometido con la inclusión del niño y la niña en la educación formal, debe realizar su tarea formativa cada vez con mayor calidad y pertinencia; tratando de construir redes con las familias y el resto de los actores de la comunidad para enfrentar mancomunadamente los múltiples desafíos que implica la violencia intrínseca. 
Un pilar fundamental de nuestro proyecto es que la escuela se transforme en la cabeza de una red comunitaria, uniendo a través de ella a todos los organismos de la comunidad (Vecinal, Centro Comunitario, Centro de Salud, Centros Municipales, Clubes, Parroquias, etc.) para resolver todos juntos problemáticas que atañen al barrio -como, por ejemplo, la violencia- y que si bien se dan en la escuela no son privativas de las misma, pero sí influyen en el proceso enseñanza/aprendizaje.

El concepto de red es un concepto vincular: red es el lenguaje de los vínculos. Las redes, tanto sean intrainstitucionales o interinstitucionales, son redes de personas. Por ejemplo puede ser la persona que está en la dirección de una institución que se relaciona con su cargo, pero no se conectan cargos entre sí, no se conectan instituciones entre sí, no se conectan computadoras entre sí, se conectan personas (Rovere, 2002). Vistas como organizaciones, las redes no son piramidales ni son telarañas, no son concéntricas, son formas de articulación multicéntricas, con un enorme grado de autonomía de cada una de las unidades, y son heterárquicas desde el punto de vista de la circulación del poder.

El concepto de red implica un proceso de construcción permanente, que a través del intercambio dinámico entre sus integrantes y con integrantes de otros grupos sociales, posibilita la potencialización de los recursos que poseen (Dabas, 1998). La tarea de consolidar redes sociales es una contribución al refuerzo de la identidad de los miembros de la sociedad, estratégica en el nivel individual y social, pues es la vía para construir una mayor integración social. O para decirlo en un sentido inverso, para reducir la marginación y la exclusión. Cuanto más densa sea la trama de redes sociales, cuanto más articuladas estén, la sociedad tendrá más capacidad para respetar las diferencias, para acceder al reconocimiento mutuo, para desplegar la solidaridad y para construir alternativas ante los problemas. Es, por lo tanto, estratégica para democratizar la sociedad y estabilizar la convivencia. Pero cada una de estas redes tiene una cierta especialización. Por ello, la participación en ellas supone también especialización de aportes y restricciones a las aspiraciones. Los atributos señalados con respecto a la participación que se observan en las sociedades complejas y heterogéneas donde vivimos, son las características de la participación en redes sociales orientadas a la resolución de problemas y a la satisfacción de necesidades. Por lo tanto, toda intervención en redes dirigida a su construcción y consolidación debe ser conceptualizada, en primer lugar y sólo como medio, como un aporte a la participación social. Más allá de este carácter instrumental, concurre al fortalecimiento de 
las identidades y a la concreción de un conjunto de valores irrenunciables: libertad, solidaridad y justicia (Martínez Nogueira, 1999)

La red se convierte en una alternativa para el desarrollo comunitario cuando en cada uno de los actores implicados se producen cambios en los niveles de las condiciones materiales de existencia y de la construcción subjetiva de la realidad. La interacción representa un aprendizaje personal, grupal y comunitario que se manifiesta en el modo de enfrentar los problemas. Un elemento constitutivo de la intervención en red es la existencia de un dispositivo que posibilita la negociación. Es por ello que las redes han de ser pensadas como organizaciones heterárquicas, como experiencias de poder compartido, de cogestión, en las cuales existe un terreno para la construcción de las reglas del juego. Otro elemento que posibilita hablar de la intervención en red es el manejo de los tiempos en escala humana: tiempos humanos posibles, que evitan el desgaste, respetuosos de los procesos personales. Es fundamental, asimismo, que se oriente a lograr acuerdo, consenso y respeto en cuanto a los plazos en las tareas propuestas y los responsables visibles.

Un problema comunitario, definido por los integrantes, es el mejor camino de acceso al crecimiento de la capacidad de autoindependencia porque su resolución hace factible la elaboración de una metodología y una tecnología propias, conformando la transformación de la persona-objeto a la persona-sujeto, a través de la autoestima lograda.

También es necesario generar una historia común, explorando todas las voces y perspectivas. Esto es, el o los problemas similares que los unen, las diferencias de origen, familiares, de intereses, laborales o educativas, las migraciones, las pérdidas, los logros. También es fundamental incluir el rastreo de los resultados obtenidos en los intentos de solucionar el problema y dónde ubican los obstáculos. Es notable comprobar cómo de la diversidad de historias y situaciones se va definiendo un problema común, que es el que le da sentido a ese estar juntos. Es entonces cuando surge la oportunidad de dar lugar al surgimiento de propuestas alternativas, posibilitando modos alternativos de describir y encontrar soluciones. Algunos autores las denominan nuevas historias y señalan algunas características interesantes a tener en cuenta: incluyen una dimensión temporal, ya que marcan aspectos de evolución y cambio, progresión y futuro; implican una actitud constructiva; es importante que se basen tanto en los ejemplos de competencia previa demostrada como en la utilización de recursos que se poseen; dan intensidad a las conexiones con otras organizaciones del contexto; contienen reglas éticas explícitas e 
implícitas, tales como respeto por los intereses de sí mismo y de los otros, evitación de la opresión y sufrimiento, sentido de responsabilidad colectiva, entre otras. (Dabas, 1998).

En otras palabras, podemos afirmar, entonces, que la metáfora de red representa un modo de interpretar la realidad. En tal sentido es un concepto teórico que invita a internarnos en los laberintos multidimensionales del conocimiento, la acción y la emoción de sujetos complejos. En un sentido más práctico y estratégico, es posible pensar en el intento de organizar estas interacciones informales de un modo más formal, proponiendo la intervención en red como alternativa para el desarrollo comunitario.

Si la escuela forma parte de una red, junto a los demás miembros de la comunidad, entre todos pueden resolver problemas comunes. Es algo muy distinto a tener relación con el Centro Sanitario del Barrio, porque la escuela envía un alumno, por ejemplo, para que lo atienda el pediatra, o en determinado momento solicita una charla sobre determinado tema a ese profesional.

\subsection{Hipótesis generales}

- La elevación de la calidad educativa, de parte de la escuela, de acuerdo con su estatuto fundacional, se corresponde con la de la calidad de vida de la comunidad.

- La construcción de redes interinstitucionales, con centro en la escuela, favorece la resolución de problemas comunitarios.

\subsection{Subhipótesis}

- La revalorización del rol docente como agente del proceso enseñanza/aprendizaje se correlaciona con la calidad educativa de la escuela

- El rol del equipo directivo es fundamental tanto para el fortalecimiento de las prácticas docentes en contextos sociales adversos, como para la construcción de la red de relaciones escuela-comunidad

- Las relaciones actuales entre los miembros de la comunidad aparecen como excluyentes, anárquicas y fuertemente centralizadas en sí mismas, lo que no permite el entramado en redes 


\section{Metodología}

El trabajo comprende varias etapas. Una primera tiene que ver con un relevam iento de información de la situación de escuelas ubicadas en asentamientos irregulares de la ciudad de Rosario. Para lo cual elaboramos un diseño exploratorio y descriptivo dirigido a generar conocimientos de tipo diagnóstico acerca de la situación real de las mismas.

Obtenidos los resultados de la primera etapa de la investigación llevamos a cabo como segunda etapa- propuestas de investigación-acción con distintos actores de las escuelas urbano-marginales de la ciudad de Rosario (elegidas como casos).

La estrategia metodológica más adecuada para esta investigación, cuya finalidad es comprender situaciones y procesos, es particularizar el problema -focalizado en el eje escuela-comunidad- y considerar la generalización al interior del caso, desde un sentido endógeno o de significado local. Esta investigación con estudios de casos persigue la intención de analizar en forma intrínseca el fenómeno de estudio, ya que queremos describir y mejorar la comprensión de los casos mismos desde la perspectiva de los actores sociales. Establecimos problemas de casos particulares, contextualizados en su comunidad.

La selección se justifica en relación con el propósito de aprender y aportar al mismo profundizando la comprensión sobre la formación integral que se imparte en el dominio de lo social, educativo, las tendencias actuales para el cambio social e institucional y la realidad de las actividades científicas en este tipo de estudios. Empleamos estrategias metodológicas cualitativas para la comprensión del caso y también utilizamos datos cuantitativos para dimensionar la población del estudio. Creemos que los problemas educativos y sociales en general, pueden y deben ser abordados, desde un punto de vista cualitativo y cuantitativo. El primero, se utiliza para posibilitar que ciertos contenidos -presentes de manera insuficiente desde la medición cuantitativa- admitan un análisis que lleve a descubrir problemas sociales, y de formación.

\subsection{Relevamiento de información primaria, que comprende dos modalidades}

\subsubsection{Relevamiento extensivo}

Obtención de información de un universo amplio, las 22 escuelas ubicadas en los asentamientos irregulares de la ciudad de Rosario, en la denominada Región VI de la Provincia de Santa Fe, por el Ministerio de Educación y, además, pertenecientes al 
Programa Integral para la Igualdad Educativa (PIIE ) del Ministerio de Educación de la Nación.

\subsubsection{Relevamiento intensivo}

Accediendo a una información detallada, con un universo de población acotado (por tiempo, y recursos), aplicado a las escuelas seleccionadas y a las instituciones de su medio. Las técnicas utilizadas para conocer las interrelaciones escuela-comunidad son las observaciones de campo y las entrevistas con las personas a cargo de la dirección de las escuelas y docentes.

Las observaciones de campo se realizan en distintas situaciones:

a- observaciones generales de las escuelas, e instituciones de la comunidad, mediante recorrido y charlas informales.

b- observaciones implementadas en las instituciones

c- observaciones realizadas en situaciones de las entrevistas.

Las entrevistas son abiertas, flexibles y atentas a la lógica del entrevistado, organizadas a partir de temas de conversación vinculados con la escuela y con el impacto de la escuela en la comunidad.

La revisión de documentos es una estrategia importante pues sirve como sustituto de los registros, cuando no se puede observar directamente. La información se refiere a documentos relacionados con antecedentes e historias institucionales, como legislaciones, protocolos, anexos ministeriales, documentos internos que se vinculen con estos tópicos, normas emanadas de organismos internacionales en esta materia, y otros, sobre el proceso de cambio social que se está llevando a cabo. La descripción de contextos relacionados con el propósito del estudio, en lo atinente a lo social, histórico y cultural, es otra estrategia en este estudio intrínseco de caso.

\section{Resultados y discusión}

\subsection{Análisis estadístico}

Con la finalidad de contextualizar la problemática de las escuelas analizadas en el marco de las escuelas PIIE de la ciudad de Rosario, antes mencionadas, se analizó la información de las mismas acerca de rendimiento y nivel socioeconómico de estudiantes, 
provista por el Sistema de Información de la Gestión Administrativa Escolar de la provincia de Santa Fe.

El objetivo del análisis fue la construcción de una tipología de escuelas mediante las técnicas de análisis multidimensional de datos (Benzécri, 1976) que permiten la visualización de la estructura de la información a través de la proyección de las variables y los individuos en los planos factoriales. La cercanía entre los puntos reflejan las relaciones entre los mismos, de manera que la interpretación puede realizarse mediante la observación de las clases o grupos formados (Moscoloni, 2005)

Los datos se procesaron mediante el sistema SPAD v.4.5 (Lebart et al, 2000) construyéndose dos matrices de datos:

\subsubsection{1ํanálisis}

Con el objetivo de detectar posibles diferencias en la estructura gradual, se consideraron las variables matrícula, repitencia y sobreedad para cada uno de los grados en cada escuela y para tres años consecutivos hasta la creación del programa PIIE.

Se construyó una matriz de escuelas-grados por matrícula, repitencia y sobreedad para cada año, se aplicó un análisis de correspondencias simples más cluster sobre coordenadas factoriales construyéndose una tipología de escuelas-grados que agrupó tres clases de rendimiento educativo:

El grupo 1 se caracteriza por un aumento en la repitencia y la sobreedad y un descenso en la matrícula en $1^{\circ}, 4^{\circ}, 8^{\circ}$ y $9^{\circ}$, son las escuelas con mayores problemas administrativos y que van perdiendo su población habitual de estudiantes.

El grupo 2 se caracteriza por una disminución en el tiempo de la repitencia y la sobreedad en $1^{\circ}, 2^{\circ}, 3^{\circ}$ y $5^{\circ}$ grados.

El tercer grupo registra un aumento de la matrícula y la sobreedad y una disminución de la repitencia, en especial en los grados: $3^{\circ}, 5^{\circ}, 6^{\circ}$ y $7^{\circ}$

Es en este grupo donde se localizan las 4 escuelas tomadas como casos, aún cuando no haya sido éste el criterio para la selección de los mismos.

\subsection{2 $2^{\circ}$ análisis}

En un segundo momento de análisis, a los efectos de poner de manifiesto posibles diferencias entre escuelas, se construyó otra matriz de datos con cada escuela como unidad de análisis resumiéndose los datos de cada una mediante los siguientes indicadores: 
- Matrícula total al último año considerado. Refleja la categoría de la escuela en función del tamaño y tiene incidencia en el presupuesto asignado. En el grupo de escuelas considerado varía entre 214 y 1288.

- Diferencia porcentual de matrícula desde el inicio al fin del período. Numerosas escuelas de este contexto pauperizado vieron disminuida su matrícula en los últimos años debido en algunos casos al traslado de niños y niñas de una clase social menos empobrecida a otras escuelas con mayor nivel socioeconómico de su población estudiantil. En este grupo todas las escuelas disminuyeron su matrícula total hasta un máximo del $50 \%$.

- Diferencia porcentual de matrícula desde $1^{\circ}$ a $7^{\circ}$ grado. Refleja el nivel de desgranamiento del establecimiento. Varía en este grupo entre 0 y $356 \%$

- Diferencia porcentual de repitientes desde el inicio al fin del período. Indicador de la calidad educativa, aunque en este contexto se relativiza su importancia debido a que la retención del alumnado es casi forzosa (importancia de mantener la matrícula, función social del comedor escolar) Los porcentajes de disminución de repitientes variaron entre 17 y $230 \%$

- Porcentaje de repitientes de $1^{\circ}$ grado. Importante indicador de calidad educativa que en este grupo de escuelas varía entre 4 y $44 \%$ (el porcentaje provincial es 6\%)

- Porcentaje de sobreedad total. Indica el porcentaje de personas con edades superiores que las relativas al grado que cursan. Varía entre 0 y $58 \%$.

- Índice socioeconómico. Coeficiente que refleja la situación familiar de la comunidad escolar en base al ingreso del grupo familiar y la ocupación de los padres. La escala de referencia es: 0-30 Bueno, 31-60 Regular, 61-100 Deficiente, siendo el coeficiente provincial igual a 69 . En este grupo de escuelas no baja de 70 .

En su conjunto estas escuelas poseen índices de rendimiento escolar muy bajos, con índice socioeconómico deficitario, con problemas de decrecimiento de matrícula, que en general han bajado el porcentaje de repitientes pero conservan un alto porcentaje en primer grado, acompañado de sobreedad y desgranamiento de $7^{\circ}$ a $1^{\circ}$.

Se presenta la tabla siguiente con el propósito de comparar los valores promedio de estos indicadores en el total de escuelas PIIE con los de las escuelas tomadas como casos (tipos E y NE descriptos en el punto 4.2) 


\section{Tabla №1}

Promedios de los indicadores en las 22 escuelas y en las escuelas casos

\begin{tabular}{|l|c|c|c|}
\hline Indicadores & $\begin{array}{c}\text { Escuelas } \\
\text { E }\end{array}$ & $\begin{array}{c}\text { Escuelas } \\
\text { NE }\end{array}$ & $\begin{array}{c}\text { Promedio } \\
\text { general }\end{array}$ \\
\hline Matrícula total al último año considerado & 460 & 726 & 643 \\
\hline Diferencia \% de matrícula: inicio a fin de período & -29 & -7 & -10 \\
\hline Diferencia \% de matrícula: $1^{\circ}$ a $7^{\circ}$ grado & -32 & -12 & -46 \\
\hline $\begin{array}{l}\text { Diferencia \% de repitientes: inicio a fin de } \\
\text { período }\end{array}$ & -134 & -169 & -122 \\
\hline$\%$ de repitientes de 1ํgrado & 23 & 52 & 28 \\
\hline$\%$ de sobreedad total & 43 & 47 & 28 \\
\hline Indice socioeconómico & 89 & 91 & 88 \\
\hline
\end{tabular}

Los indicadores reflejan problemas estructurales en las 4 escuelas seleccionadas como casos. En un análisis exclusivamente cuantitativo estos indicadores estarían mostrando en las escuelas de tipo $E$ (ver punto 4.2) una peor situación en cuanto a matrícula y desgranamiento debido a las características de este contexto ya señaladas. Sin embargo, el análisis cualitativo permitió desde otro ángulo, la consideración de otros aspectos positivos en estas escuelas, debido en parte a la acción del equipo directivo.

\subsection{Estudio de casos}

De la totalidad de las escuelas PIIE, seleccionamos -por observación directa- cuatro escuelas casos, en base al cumplimiento de diferentes roles directivos. Nos concentraremos en describir y comprender la perspectiva de los distintos actores sociales de los cuatro casos seleccionados. Pretendemos conocer lo que implica el caso en relación con el aspecto vinculación entre el mundo educativo y su comunidad y descubrir los temas entramados en la acción de esa vinculación.

Todos sabemos que actualmente muchos equipos directivos tienen dificultades para ejercer la dirección de los centros educativos. Al llegar a los respectivos cargos, se encuentran con una trama compleja, estabilizada al amparo de una tradición convertida en cultura organizativa, que les dificulta ejercer las responsabilidades asumidas. La hipertrofia reglamentarista de los diferentes rasgos que inciden sobre la vida y tareas escolares dificultan la implicación del equipo directivo en la marcha de la escuela. Uno de los efectos no deseados de esta hipertrofia es que, ante el riesgo de que cualquier oscilación sobre el marco normativo genere un conflicto, los directores y directoras circunscriben sus 
actuaciones a lo estrictamente regulado. Es más seguro trabajar sobre lo reglamentado que entrar en el terreno vidrioso de lo creativo y pedagógico en el que todo puede ser cuestionado.

Dos de las instituciones (E1 y E2) responden a este estilo directivo, donde una cabeza conduce, ordena, cumple las tareas de índole burocrática-administrativas, pero también la de relación con los demás miembros de la comunidad y la gestión de los asuntos pedagógicos. Por lo cual, externamente, se observa orden en el trabajo, puntualidad, limpieza, disciplina.

El tercer caso (NE3), también como en los dos anteriores, la Dirección es asumida en julio del 2006, o sea tiene relativamente poca antigüedad en la función. Pero, a diferencia de los mencionados, todavía no logra acordar criterios con las vice-directoras, algunas de las cuales tienen antigüedad en la institución. Percibiéndose un malestar que se trasluce en docentes de los distintos turnos. Hay enfrentamientos internos a nivel de equipo directivo y de docentes, lo cual conduce a indecisiones, imprecisiones en la forma de abordar la tarea común. Pero se percibe, desde la Dirección, una clara idea de superar los problemas y avanzar 'con todos', hacia la tarea común. El cuarto caso (NE4), se caracteriza por falta de directivas, enfrentamientos en el equipo de conducción, una apatía generalizada ante la situación del alumnado y una toma de conciencia de que 'acá no se puede hacer nada', lo cual es bastante paralizante, por un lado y consecuentemente improductivo desde la óptica del proceso enseñanza / aprendizaje.

En ningún caso encontramos un estilo que se aproxime a lo mundialmente requerido, o sea:

Lo que ahora se le demanda es: activar comportamientos colegiales, procurar el desarrollo de la profesionalidad docente de los profesores y posibilitar el liderazgo compartido; supervisar el desarrollo del curriculum y las instrucciones generando debates públicos y las acciones consecuentes del centro escolar (Llavador, 1997, p.132)

En otras palabras, no nos hemos encontrado con directores o directora que no sólo atiendan la tupida trama de regulaciones, sino además armonicen los intereses de los distintos actores en pos de la construcción consensuada y en conjunto de la comunidad educativa. 


\subsubsection{Trabajo de campo en las escuelas}

Una vez seleccionados los casos, se procedió a realizar la entrada a campo, mediante la observación participante. Obtuvimos así una primera visión general de cada escuela y de su inserción en la comunidad barrial.

Cuando no se pudo observar directamente en la magnitud deseada, sirvió como sustituto la revisión de documentos acerca de las escuelas de referencias. Esta estrategia fue importante pues usamos registros tales como información relacionada con antecedentes e historias institucionales, documentos internos vinculados con estos tópicos, normas de la institución y otros.

La necesidad de profundizar en las perspectivas de los actores sociales participantes de la problemática, educadores, estudiantes, funcionarios y funcionarias gubernamentales, tiene como horizonte el análisis, el diálogo y la integración a este proceso de generación de conocimiento. Paralelamente se realizaron entrevistas a las direcciones y docentes de las escuelas-casos, se buscó información a través de la observación directa para ver cómo se manifiestan las personas en estudio, preservando las realidades múltiples, las visiones diferentes o incluso contradictorias con las que nos encontramos. Para facilitar este estudio, hemos aproximado una organización conceptual, ideas que expresan la comprensión que se necesita, puentes conceptuales que se inician desde lo que ya conocemos y descripciones que guían la recolección de datos. Para este cometido, se elaboraron los ejes para su aplicación en entrevistas no estructuradas.

En relación con los docentes, se elaboró otro instrumento de recolección de datos (cuestionario), dejado en cada institución para ser respondido voluntariamente. La importancia de esta estrategia metodológica radica en conocer la perspectiva del actor social más importante implicado en la institución, respecto del vínculo entre las esferas comunitarias y el mundo educativo.

\subsubsection{Análisis e interpretación de las entrevistas}

Las entrevistas a los directores y directoras han sido en profundidad, si bien se convinieron los temas que no debían faltar, esto no significó llevar preguntas elaboradas a priori. Nos interesó la narrativa de cada persona, dejando la exposición libre interviniendo sólo si no emergían los temas que habíamos convenido. Pensamos en estas entrevistas etnográficas como una serie de conversaciones en las que introdujimos lentamente nuevos elementos para ayudar a los informantes a responder. 
En el análisis de la interpretación de textos hemos considerado la codificación del material con el propósito de categorizar. La codificación abierta nos ha llevado a desenmarañar los datos clasificando las expresiones por sus unidades de significados. La interpretación de estas narraciones según ya hemos establecido ha arrojado las siguientes categorías:

\subsubsection{Iniciativas Pedagógicas (I.P.)}

Los directores y directoras de las cuatro escuelas hacen referencia a las I.P. como Proyectos Institucionales, implementados exitosamente por los docentes. Sin embargo, sabemos -según surge de las otras fuentes de información- que en todas las escuelas estas expresiones no son reales.

Algunas I.P. fueron construidas a partir de un diagnóstico acerca de los problemas existentes.

...Elaboramos un Proyecto de Juego y convivencia, porque cuando esta Directora y la Vice llegamos a la escuela, sentimos que las relaciones docente-niño, docente-padres, estaban cargadas de agresividad, el contacto era la violencia entre unos y otros y el reto de los docentes. Comenzamos a indagar cuáles eran los momentos más difíciles en cuanto a la disciplina...Los alumnos señalaron que no les gusta que les griten y les gusta la escuela porque se sienten seguros. (E2)

En otros casos, las respuestas acerca de las I.P. son vagas e imprecisas.

...La IP abarca acciones en Talleres, trabajo en biblioteca, para todos los grados, por ejemplo "Maratón de Lectura" y huerta (que está todavía en proyecto). En este aspecto también podemos hacer alusión al uso de la sala de computación, a la murga y a los juegos. (NE4)

Como queda claro, en la elaboración de ninguna I.P. se tuvo en cuenta la relación con alguna institución de la comunidad. Es evidente por ejemplo, que el tema de la violencia escolar es una problemática social, que como tal tiene que ser encarada por toda la comunidad y que la escuela no puede lograr la solución unilateral y aisladamente.

\subsubsection{Materiales didácticos provistos por Programas Especiales}

Los discursos directivos muestran una diferencia abismal entre los dos grupos de escuelas. En las escuelas $E$ los directores o directoras manifiestan un reconocimiento de los materiales enviados por el Plan Social y actualmente por el PIIE, tanto en su cantidad, variedad, como Volumen 9, Número 1, Año 2009, ISSN 1409-4703 
la calidad de los mismos. Además demuestran una verdadera preocupación para que los docentes utilicen de manera eficaz los recursos, en el dictado de sus clases. En las escuelas $\mathrm{NE}$, en cambio, mencionan algunos aisladamente y no dan cuenta del uso por parte de sus docentes, o aluden a que los maestros y maestras desprestigian e invalidan el material, por la falta de pertinencia al contexto.

...El PIIE ha aportado muy buenos materiales. A través del PIIE la Escuela ha recibido juegos para Lengua y Matemática... Con el Trengania, una docente realizó todo un proyecto áulico muy bueno, valorado hasta por los padres. (E2)

...Libros para 1ro. y 2do años, libretas para 1ro., guardapolvos. Los materiales bibliográficos y didácticos no se usan, porque "no responden a la realidad de los alumnos", por ello los docentes "no se engancharon" prefieren usar los que ellos ya usan o construyen. "Los maestros no lo utilizan". El material llega fraccionado y algún material no llegó completo. (NE4)

Los directores y las directoras, aún los que reconocen la importancia de los materiales para la escuela, ni siquiera vislumbran la posibilidad de obtener un aprovechamiento mayor, usándolos como pieza de intercambio con servicios que puedan brindar instituciones del barrio.

\subsubsection{Infraestructura -Sala de computación}

En las cuatro escuelas existe al presente la Sala de Computación totalmente equipada, así lo aseveran sus directores y nosotros lo comprobamos en nuestro trabajo de campo. Cuentan con servicio de alarma, por los robos reiterados. La diferencia es que en las escuelas E, las ocho PC y las cuatro impresoras están conectadas y en las restantes las PC no están en red, funciona una sola impresora y las demás continúan embaladas.

...La Sala de Informática está funcionando. Llevan sus alumnos aquellos docentes que saben informática. (E1)

...La Sala de Computación existe, está instalada, pero no se usa, porque los docentes no saben computación. (NE4)

El Ministerio de Educación Provincial brindó un curso de PC básico al cual pudieron asistir un grupo reducido de docentes, además de ampliar este espectro es importante brindar Internet a las escuelas, para que la población estudiantil pueda buscar información. Pero lo que no realiza o no tiene los medios para realizar el nivel central, lo podría hacer la escuela. 
Mediante una red comunitaria, se podrían ofrecer servicios a cambio de que otra institución brinde conocimientos de informática y/o solvente el abono a un servidor.

\subsubsection{La capacitación docente}

En esta categoría los discursos son coincidentes: en las cuatro escuelas no hubo capacitación docente de ningún tipo. Sin embargo, debemos destacar que algunas directoras supieron resolver por sí mismas este problema de manera creativa.

Debemos resaltar que la capacitación más requerida es en el área de computación, debido al enorme avance tecnológico.

...Como problema y como deficiencia del PIIE, el hecho de no contar con personal que capacite a las docentes para usar las máquinas de manera relacionada con las actividades de aula. A través de la Cooperadora le pagamos a un Coordinador de Área para trabajar con los chicos y docentes, de manera que aprenden todos juntos. Para capacitar al docente en promoción asistida, lo hacemos nosotras a partir de la bibliografía disponible. (E2)

...Solicitamos capacitación para el uso del material del PIIE, lo que permitiría el conocimiento y el uso didáctico. Capacitación en particular en computación, no hemos tenido "master teacher"; a pesar de ello los maestros, por propia iniciativa, hacen uso de las PC. (NE4)

\subsubsection{Construcción de redes}

Los directores y directoras coinciden en que es una tarea que les falta realizar, la construcción de redes comunitarias, con organismos existentes en el barrio. En algunos casos hubo intentos muy positivos, pero fracasaron. En otros se confunde, la mera relación de invitar a un especialista a exponer un tema, o enviar un alumno a los Centros Asistenciales, con la construcción de verdaderas redes, para solucionar mancomunadamente los problemas barriales.

....No he hecho redes porque sé las limitaciones que tenemos, es complejo sostener la escuela en su interior, sí tengo relaciones con instituciones como un Centro de capacitación que funciona muy bien y al que mando a aquellos chicos que están de alguna manera varados aquí, sin poder avanzar, porque la escuela no puede ofrecerles lo que necesitan (E1)

...No tenemos experiencias de relaciones con Centros de la comunidad, nada más que esporádicamente con el Centro Crecer y con Psicólogos y Odontólogos del Centro de Salud, a los cuales derivamos algunos alumnos. Como organismos reconocemos la exítencia del Volumen 9, Número 1, Año 2009, ISSN 1409-4703 
Club y de una Vecinal (que no cumple ninguna función). Por problemas de luz y agua en el Barrio, intentamos hacer reuniones con todos los vecinos para realizar pedidos comunitarios ante las respectivas entidades, pero fracasamos. (NE3)

Al formular esta cuestión en las entrevistas queda claro que las directoras desconocen la conformación de redes comunitarias y aunque los entrevistadores les explican el concepto básico de integración para la resolución de problemáticas comunes, responden de acuerdo con su imaginario que es siempre la relación unilateral y con un objetivo preciso.

\subsubsection{Biblioteca}

El decir de las personas directoras varía entre las escuelas $E$ y las escuelas NE. En las primeras reconocen la gran importancia de la infraestructura para las bibliotecas, enviadas por los Programas, al igual que los libros y fomentan el uso intensivo de las instalaciones. Los segundos mencionan la existencia de la Biblioteca y reconocen como muy importante el envío de libros, pero no llevan a cabo acciones para generalizar y facilitar su uso.

...Dos bibliotecas móviles, una con libros para el segundo y tercer ciclo y la otra con libros para el primer ciclo. Con el dinero del PIIE compramos para la Biblioteca seis mesas y sillas. Los alumnos asisten a la Biblioteca acompañados de sus docentes. Una persona que efectúa tareas diferentes se encarga de catalogar los libros. Los libros del primer año, que llegan todos los años, se entregan a los alumnos para que los lleven a sus casas. (E2)

...Contamos con biblioteca, sin bibliotecario, por ello la biblioteca no se usa.(NE3)

Con todo este material, podría surgir desde la escuela la generación de puntas de red, generando distintas acciones, entre otras: brindando lectura a los padres, abriendo la biblioteca a otros organismos de la comunidad, posibilitando la lectura del periódico del barrio, etc.

\subsubsection{Procedencia de los alumnos}

Las autoridades de las cuatro escuelas confirman que sus estudiantes proceden de los asentamientos irregulares, salvo algunas pocas excepciones. Sostienen que son educandos con problemas estructurales, tanto socio-económicos como culturales. Sin embargo, las opciones pedagógicas que sostienen los directores y directorass para resolver las dificultades que presentan estos estudiantes, son disímiles. Mientras que las escuelas $\mathrm{E}$ se inclinan por el sí se puede, las otras se enrolan en el no se puede. 
...Los alumnos provienen todos del asentamiento marginal, a la mañana hay gran porcentaje de inasistencia porque los padres no los despiertan (NE3)

...La matrícula está compuesta con muchos migrantes del Chaco. Hay marcada diferencia entre los alumnos del turno mañana y del turno tarde. Los primeros son del barrio y los padres se ocupan de ellos, asisten a las reuniones, aunque no hay actividad con los padres. Los alumnos de la tarde son los del asentamiento irregular de atrás de la escuela. (NE4)

\subsubsection{Ocupación de los padres}

La ocupación de los padres también es similar en los cuatro casos, o están desocupados, o viven de la recolección y venta de residuos reciclables (cirujeo, cartonero) y unos pocos trabajan en la construcción.

...Los padres son cartoneros o cirujas y llevan a los chicos a trabajar con ellos en los carros (E1)

... Toda la familia trabaja del cirujeo, incluido los chicos (NE3)

...Algunos padres trabajan de peón de albañil, y los otros son cartoneros. (NE4)

\subsubsection{Inclusión}

En las escuelas E, se evidencia una manifiesta preocupación de los directivos por mantener los alumnos en la escuela, lo cual ya es sumamente importante. Ello no ocurre en las NE, donde existe preocupación por la deserción, pero basada más -según nuestro entender- en el temor por la disminución de la matrícula que por el interés de la permanencia real de los niños en la escuela.

...Otra cuestión que preocupa a esta directora es el deseo de que los chicos continúen estudiando, incluso más allá de las instituciones de la zona, para capitalizar sus capacidades.

También estoy preocupada por la apertura de la escuela a la comunidad y la participación de los padres, cuestión que antes no ocurría. Muchos son hijos de analfabetos. (E2)

...Hay mucha deserción, porque los padres entienden como una manifestación de progreso social, cambiarlo de escuela, mandarlo a otra que no sea del Barrio, aunque sea otra escuela pública, ubicada en otro asentamiento marginal, y hasta tengan que pagar transporte. (NE3)

Observamos que en algunos casos existe preocupación "por la apertura de la escuela a la comunidad" a través de la participación de los padres. Sin embargo se descuida el hecho de que la participación no se logra exclusivamente por invitaciones de la escuela. Los Volumen 9, Número 1, Año 2009, ISSN 1409-4703 
padres reconocen a la institución cuando la misma tiene un reconocimiento del barrio, cuando se gana ese lugar en base al trabajo en la comunidad y con las instituciones de la misma.

\subsubsection{Entrevistas a docentes}

Utilizamos las mismas categorías de análisis antes enunciadas. Las entrevistas a docentes fueron semiestructuradas en cuanto, a partir del decir de los directores y directoras, de la entrada a campo y de lo que habíamos propuesto como evaluación ya teníamos algunas preguntas, de cualquier manera nos interesó que el relato fuera fluido.

Las docentes entrevistadas fueron seleccionadas mediante la técnica de informantes claves en red. Una vez logrado el acceso a campo en la institución, nos enfrentamos a la tarea de cómo llegar a las docentes, nos interesó realizarla con aquéllas que voluntariamente se ofrecieron y a las que éstas invitaban a su vez.

\subsubsection{Participación de las docentes en Programas Nacionales o Provinciales}

Quisimos conocer, en primer lugar, el interés y la posibilidad de las docentes de participar en Programas -como el Plan Social y actualmente el PIIE- que subsidian proyectos de mejora educativa.

Las respuestas quedan claramente diferenciadas según los casos extremos. De cualquier forma no hay homogeneidad en las situaciones de cada escuela, mientras que en la E1 se resalta que:

...Participa desde el comienzo de la experiencia. La Iniciativa Pedagógica (I.P.) que participa del Programa PIIE comenzó como un Proyecto circense desde el que se trabajaba sobre inventos y experiencias científicas. La Directora de la escuela planteó esta experiencia en una reunión plenaria, como disparador para plantear la I.P., y también la ventaja de participar en el Programa PIIE.

En la E2 no hay un relato explícito acerca de esta categoría. Por otro lado, las docentes entrevistadas de las otras dos escuelas coincidieron en expresar su escasa participación. De los mismos se desprende que en la NE3 participaron solamente dos docentes y en la NE4 una. Son comunes escuchar en estas dos escuelas, expresiones como:

...No participo en el Proyecto, no conozco su desenvolvimiento. Me entero de su funcionamiento, sintéticamente, en las Reuniones Plenarias, y también porque me invitan a Volumen 9, Número 1, Año 2009, ISSN 1409-4703 
participar escribiendo para la Revista. El dinero del PIIE se invirtió todo para la edición de la Revista del año pasado. (NE3)

...Conozco el PIIE, pero no trabajo. Es un Programa muy bueno, porque dieron mucho material. Los docentes trabajan en la Revista de la escuela, me piden que lleve artículos...Otro Proyecto es el de microemprendimientos, para el 3er. Ciclo. (NE3)

\subsubsection{Iniciativas Pedagógicas}

Como en la categoría anterior, las polarizaciones se hacen notorias. Mientras que no hay respuestas de trabajo concreto en las escuelas ni en el aula en las NE3, NE4; en las E1 y E2 las docentes expresan su participación en la I.P. y se nota entusiasmo en la implementación de la misma en la escuela.

... Trabajo desde la transversalidad de los conocimientos, especialmente con lengua. Trabajo por ejemplo con biografías de científicos reconocidos. (E1)

...Los proyectos escolares incluyen temas variados: convivencia y juego, lectura, música e integración con alumnos con promoción asistida de primero, segundo y tercero. Los dos primeros son Proyectos Institucionales y como tales abarcan a todos los docentes y alumnos, los otros responden a agrupaciones parciales de docentes. (E2)

De parte del equipo docente, todo gira en participar o no en la IP pensada desde la Dirección de la escuela, pero no se percibió en las entrevistas ninguna sugerencia sobre organizar la Iniciativa con las instituciones de la comunidad, ni siquiera para encarar las problemáticas que afectan diariamente el normal desenvolvimiento de la clase.

\subsubsection{Materiales didácticos provistos por Programas Especiales}

En general, las docentes de las cuatro escuelas pueden dar cuenta que llegó material del PIIE a sus instituciones; la diferencia radica en que mientras unas precisan detalladamente todo el material recibido (E1 y E2), otras mencionan vagamente la existencia de alguno de ellos (NE3 y NE4).

...Se enviaron libros nuevos, para una biblioteca que sólo tenía unas pocas obras viejas, se organizó el material recibido, fue un objetivo común que ayudó a hacer coincidir a las docentes trabajando en conjunto en torno a un proyecto educativo. (E2)

Debemos resaltar que las docentes entrevistadas se preocuparon por dar cuenta detallada, en las escuelas E1 y E2, de cómo usaron los recursos enviados por el PIIE. 
...En la escuela se usan todos los recursos recibidos. Los libros se utilizan tanto en la biblioteca como en el aula. Incluso se ha establecido un sistema de préstamo que favorece que los alumnos se lleven los libros a sus hogares. En lo personal utilizo también en las clases los CD con cuentos para niños y el material para Matemática (E2)

...Para esto utilizo el material bibliográfico del PIIE, realizo visitas y trabajo de campo. En $6^{\circ}$ grado el tema es el aire. Utilizo los aparatos y el material de video adquiridos gracias al PIIE y otros programas. Utilizo mucho y aprovecho la diversidad de manuales de que disponen lo que me permite analizar con los chicos diferentes perspectivas sobre un mismo tema. (E1)

Las docentes de la escuela NE3, si bien hacen mención al uso de material bibliográfico, desconocen el resto de los materiales enviados por el PIIE.

... Uso TV, DVD, no uso los recursos didácticos. Los docentes del 1er. Ciclo hicieron un curso para usar el trengania. Yo no sé usar esos nuevos recursos que llegan. Respecto a los libros, ayer llegaron los del 2do. Ciclo, muy buenos y de editoriales muy conocidas. (NE3)

Las respuestas de los docentes muestran la riqueza del material recibido en las escuelas y aún cuando en un caso resaltan la importancia de que los "alumnos se lleven los libros a sus hogares", ninguno sugiere la intervención de estos materiales en proyectos comunitarios.

\subsubsection{Infraestructura - Sala de computación}

Esta categoría aparece como una de las más conflictivas, debido a varios factores: tales como falta de infraestructura en la escuela, envío de las PC, desconocimiento de su manejo de parte de las docentes, falta de capacitación, entre otros. Pero, por contrapartida, aparece como una de las más valoradas, pues cuando realizamos el trabajo de campo fue uno de los lugares predilectos de las autoridades de las escuelas para mostrarnos como un logro institucional.

Las opiniones varían entre las docentes entrevistadas, desde las que hacen una breve mención al aspecto externo de la Sala, hasta las que realizan hincapié en su uso didáctico. ...La sala es amplia, con abundante luz natural al igual que las dependencias anteriores. Su pintura luce impecable. Posee rejas provistas. (E1)

...La sala de computadoras despierta mucho interés entre los alumnos. No obstante, señala la carencia de material experto. Hice el curso de INTEL, pero considero que si bien es de calidad, resultó demasiado breve para quienes no tenían formación informática previa (E2) 
Acerca de la infraestructura informática, son claros los señalamientos negativos de los/las docentes, pero no existe ninguna idea relacionada con posible búsqueda de colaboración de alguna institución barrial. Idéntica situación se reitera con respecto al ítem de la capacitación docente.

\subsubsection{La capacitación}

Según los discursos de los docentes, aparece como una de las acciones más débiles y más demandadas. El Curso de Capacitación "Masters Teachers" no fue implementado para todas las docentes, siendo ésta una de las quejas recurrentes, teniendo en cuenta que la mayoría de ellas hace un uso muy limitado de la PC, debido a su falta de conocimientos.

...Reitero nuevamente la solicitud de mayor capacitación. Además del curso de Intel, asistí a una jornada sobre juegos. (E2)

...Capacitación no hubo. Yo hice por mi cuenta cursos de: Lengua, Geografía, Ciencias Naturales, acerca de la enseñanza, por supuesto y con el sacrificio de cada una. (NE3)

\subsubsection{Construcción de redes}

Si bien es, para este tipo de escuelas, una necesidad implementar estos vínculos, la acción dista mucho de haberse logrado, de acuerdo con el decir de los docentes entrevistados. En algunos casos, se apoyan en que no existen organismos en el barrio con los cuales relacionarse. Situación que nos parece extraña y que no condice en su totalidad con lo observado en nuestro trabajo de campo. En otros casos, nombran innumerables y variadas instituciones, pero sin dar cuenta de la construcción de redes comunitarias, sino únicamente la participación en algunas acciones con padres, profesionales. Asimismo, mencionan la prestación de algún servicio de odontología, o psicológico a la población estudiantil, por parte de alguna institución barrial. La realidad es que ninguna de las cuatro escuelas construyó redes comunitarias; aceptándolo ellas mismas como un déficit.

...En la comunidad existen: La Vecinal, el Club, dos dispensarios (uno Municipal y otro Provincial). A los dos dispensarios la escuela deriva chicos al Gabinete Psicológico. Con la Vecinal, la escuela se reúne para solicitar cosas, por ejemplo, ante la falta de luz, agua, transporte urbano que entre al barrio. Con el Club en verano hay un proyecto municipal para que los chicos asistan a la pileta; la escuela solamente informa de las actividades del Club. (NE3) 
...Sí, hay un Centro Crecer, una salita de emergencia, Jardín №xX y una Escuela Especial. No hay proyectos con otras escuelas, pero se empezó a tener alguna relación con esta última, integrando alumnos con necesidades especiales, para ver qué se puede hacer, con una maestra integradora de la escuela especial. Hay poca participación de los padres, no hay actividades para hacer con los padres. Los padres aceptarían y sería muy factible. Hay comedores comunitarios en el Barrio, y una Vecinal. (NE3)

En términos generales, de las entrevistas se deduce que los docentes son más reticentes aún que los directores o directora a establecer relaciones con la comunidad, o tal vez no perciben las ventajas de las mismas, señalando sólo casos muy puntuales, los cuales no forman parte de una verdadera construcción de red comunitaria.

\subsubsection{Biblioteca}

Se resalta la incorporación de material bibliográfico y de las bibliotecas, tanto fijas como ambulantes, en las cuatro escuelas. En cuanto al uso de los libros, en todos los casos se trabaja con ellos en la escuela; además, en otros, los alumnos y alumnas pueden llevarlos a sus casas.

En cuanto a la sala de Biblioteca, una de las escuelas no la posee y las docentes guardan los libros en armarios con llave en cada aula. Ninguna de ellas posee el cargo de bibliotecaria, variando la situación de la persona a cargo de la Biblioteca. Respecto a este último tema, los reclamos de docentes y autoridades son iguales en las cuatro escuelas.

...Yo uso los libros, que son muy buenos. La Biblioteca fue desmantelada, no tenemos Biblioteca. Cada maestro tiene en el salón armarios con llaves, donde guarda sus libros. (NE3)

\subsubsection{Inclusión}

Los contextos en los cuales se hallan insertas las cuatro escuelas son similares, sin embargo en las escuelas $E$ se percibe cierta esperanza y voluntad de resolver los problemas en forma positiva. Por el contrario, en las escuelas NE reina el "no se puede" y una percepción negativa con respecto a la superación de sus estudiantes.

...Respecto de las estrategias para la inserción y la reinserción escolar, considero que están desde que están los docentes en la escuela. En la escuela se realiza un verdadero seguimiento del alumno que no quiere o no puede asistir a la escuela (se lo llama, se habla con los papás, etc.). Se hace también un seguimiento de los chicos con extraedad, se los Volumen 9, Número 1, Año 2009, ISSN 1409-4703 
deriva a un centro de educación y después se lo sigue para ver si asisten o no a la escuela. (E1)

...En realidad se ve a los niños más incentivados, pero eso no significa que haya menos inasistencias. Lo que sí se percibe como fundamental es el hecho de que la escuela dé de comer, eso sí atrae y retiene niños. No obstante lo cual, señalo que han debido hacer campañas o publicidad para que los niños de la villa se inscriban en la escuela, porque la matrícula no crece. Considero que se puede estimar que están afectados de sobreedad, un $30 \%$ de los alumnos. No hay mucho desgranamiento. (E2)

...Hay mucha deserción. Yo sugerí realizar un "espacio - puente" en contraturno, para la inclusión de chicos. Empezar a trabajar en talleres y cuando el alumno estaba enganchado, llevarlo al grado correspondiente, hacer del taller "el puente". La escuela inclusiva tiene que aceptar chicos con problemas de conducta, pero no me lo aceptaron. (NE3)

...Hay mucha deserción, hay dos compañeras que están en un Proyecto de Reinserción, una es Psicóloga. Los chicos faltan mucho, más a la mañana porque no se levantan -los padres no los despiertan, porque se durmieron borrachos- porque les faltan zapatillas, ropa, de todo. Yo conozco la vida de cada uno. Cuando hay dejadez de la madre y falta de interés del alumno no se puede hacer demasiado, por lo tanto yo lo dejo. Si el chico se interesa y se esfuerza entonces lo ayudo, pero son los casos menores. (NE3.)

Con respecto a la inclusión de los alumnos, los docentes creen poder resolver la problemática -de una manera o de otra- desde la escuela. No perciben que los problemas por ellos enunciados: comedor, ausencias reiteradas, dejadez de los padres, etc., así como las soluciones ofrecidas: citación a los padres, espacio-puente, proyecto de reinserción, etc., necesitan un tratamiento integral y en conjunto por los organismos oficiales y privados. La escuela, unilateralmente, descontextualizada de la comunidad barrial, no puede realizar nada perdurable

\section{Conclusiones}

Los resultados del análisis estadístico, en concordancia con la primera hipótesis general, confirman los rasgos distintivos de las escuelas PIIE en cuanto a pauperización del alumnado y bajo nivel en los indicadores cuantitativos generales de rendimiento educativo, encontrándose diferencias de nivel entre estas escuelas. Sin embargo estas diferencias 
podrían responder tanto a disparidades en la calidad educativa, como a la movilidad de su población de estudiantes, característica de este contexto socioeconómico.

La diferenciación puede percibirse más claramente mediante el análisis cualitativo a través del cual es posible además acercar evidencias acerca de la segunda hipótesis general y de las subhipótesis, marcando las diferencias en cuanto a los roles docente y del equipo directivo y sus relaciones con los miembros de la comunidad.

En base a los estudios focalizados en las escuelas-casos, podemos decir que pese a que las cuatro escuelas reciben alumnos en situación de vulnerabilidad social, la actitud del equipo directivo y docente frente a los mismos es totalmente diferente. En las escuelas E1 y E2 la referencia a sus estudiantes y a sus necesidades aparece como una meta siempre posible de alcanzar. Esta actitud de compromiso con la comunidad permitiría pensar que estos alumnos están en condiciones de mejorar su calidad educativa y por ende su calidad de vida. En las otras dos escuelas, predomina en los discursos la ausencia de vocablos aglutinantes; no se expresa un 'nosotros', sino por el contrario es insistente la explicitación del 'yo' y el 'no puedo'. El 'no' parece reflejar un estado de mimetización con los 'no' que estigmatizan la realidad social, económica y cultural en la que están insertos los alumnos y las alumnas. Ningún discurso reconoce que el 'podemos' es más sencillo de realizar desde una comunidad barrial organizada, donde se planteen problemáticas comunes que se tiendan a solucionar mediante los aportes de todos los organismos de la red y no desde la escuela como ente aislado.

La importancia del rol docente como agente del proceso enseñanza/aprendizaje, tal como se expresa en la primera subhipótesis, se pone de manifiesto especialmente en la capacitación docente y en la utilización de los materiales didácticos y la infraestructura informática.

En relación con la primera los discursos son coincidentes, no hubo capacitación de ningún tipo. Sin embargo, debemos destacar que algunas directoras supieron resolver el problema en forma creativa. La capacitación más requerida es en el área de computación, por el enorme avance tecnológico. El perfeccionamiento docente redunda en el desarrollo de estrategias didácticas que, como es lógico, permiten la adquisición de una mayor calidad educativa. Si embargo, el mismo debiera insertarse en el contexto comunitario para así favorecer la construcción de redes.

En cuanto al aprovechamiento de equipos didácticos, actualmente las escuelas cuentan con algunos provistos por el PIIE y otros comprados con dinero aportado por el Volumen 9, Número 1, Año 2009, ISSN 1409-4703 
Programa. Las docentes en general pueden dar cuenta del material; sin embargo mientras en las escuelas E1 y E2 precisan detalladamente todo el material recibido, en las otras mencionan vagamente la existencia de alguno de ellos y desconocen su uso. En muchos casos, las compras se han realizado indiscriminadamente, respondiendo a las necesidades de la IP o a requerimiento de alguna docente aislada. No hay un aprovechamiento integral de los recursos hacia el interior de las escuelas y mucho menos de manera interinstitucional. Posibilidad esta última que se desconoce, al no integrarse a una red constituida por diferentes instituciones del barrio.

Los discursos directivos son totalmente distintos entre las escuelas. En algunas, as autoridades manifiestan un reconocimiento de los materiales enviados por el PIIE, tanto en su cantidad, variedad, como la calidad de los mismos y demuestran una verdadera preocupación para que las docentes los utilicen de manera eficaz en el dictado de sus clases. En otras escuelas, en cambio, mencionan algunos aisladamente y no lo muestran a sus docentes, o los desprestigian e invalidan su uso. Estos hallazgos permiten aportar a la segunda subhipótesis acerca de la importancia del rol del equipo directivo para el fortalecimiento de las prácticas docentes.

Concretamente en lo relativo a construcción de redes comunitarias los directores y directoras coinciden en que es un deber que les falta realizar. En algunos casos hubo intentos muy positivos, pero fracasaron. En otros se confunde la mera relación de invitar a un especialista a exponer un tema, o enviar un alumno a los Centros Asistenciales, con la construcción de verdaderas redes, para solucionar mancomunadamente los problemas barriales. Las docentes entrevistadas, en algunos casos, se apoyan en que no existen organismos en el barrio con los cuales relacionarse. Situación que nos parece inexacta y que no condice en su totalidad con lo observado en nuestro trabajo de campo. En otros casos, nombran innumerables y variadas instituciones, pero sin dar cuenta de la construcción de redes comunitarias, sino únicamente la participación en algunas acciones con padres, madres, profesionales.

La realidad es que ninguna de las escuelas construyó redes comunitarias; aceptándolo como un déficit. Es posible que la utilización excesiva del término 'red' haya producido un vacío de significación en el mismo.

Se evidencia una manifiesta preocupación de los directores y las directoras por mantener sus estudiantes en la escuela, lo cual es sumamente importante. Ello no ocurre en otras, donde existe la preocupación por la deserción, pero basada más -según nuestro Volumen 9, Número 1, Año 2009, ISSN 1409-4703 
entender- en el temor por la disminución de la matrícula, que en la permanencia real de a niñez en la escuela.

Los contextos en los cuales se hallan insertas las cuatro escuelas son similares, sin embargo en algunas se percibe cierta esperanza y voluntad de resolver los problemas en forma positiva; si bien no construyeron redes con los organismos de la comunidad, al menos intentaron algunas relaciones unilaterales. Por el contrario, en otras escuelas reina una percepción negativa con respecto a la superación de los alumnos. Creemos que esta percepción podría modificarse a través de un proceso de conocimiento y comunicación entre la escuela y las instituciones de la comunidad.

En síntesis, pensamos que el rol de directores, directoras y docentes aparece como central para la calidad educativa, en tanto hemos detectado que en los mismos contextos de pobreza hay escuelas que -dada la preocupación de dichos actores- pueden elevar la calidad educativa de sus alumnos y alumnas; mientras que en otras, la convicción de que toda iniciativa en este contexto está destinada al fracaso, invalida todo proceso de calidad educativa y por ende de calidad de vida. El hecho de que no se hayan detectado intentos de construcción de redes interinstitucionales deja a la escuela como una institución solitaria, para afrontar las problemáticas actuales del contexto socio-cultural de los alumnos y alumnas.

\section{Referencias}

Abramovay, Mario. (2005). Violencia en las escuelas: un gran desafío. Revista Iberoamericana de Educación, (38), 26-39.

Benzécri, Jean Paul. (1976). L’Analyse des données, T.I La taxonomie T.II L'Analyse des correspondances. París, Francia: Dunod.

Dabas, Elina. (1998). Red de redes. La práctica de la intervención en redes sociales. Buenos Aires, Argentina: Paidós.

Lébart, Ludovic, Morineau, Alain et al. (2000). Système SPAD, Versión 4.51. París, Francia: BCISIA-CERESTA

Llavador, Beatriz. (1997). Tradición y cambio en la dirección escolar de Francia. En De Aquí y de Allá. Textos sobre la Institución Educativa y su Dirección (pp.121-134). Buenos Aires, Argentina: Kapelusz. 
Lugo, Maria Teresa. (2002). Escuelas en innovación. El desafío de hornear el pastel del cambio. En: Cómo planifican las escuelas que innovan (pp.40-53). Buenos Aires. Educación Papers Editores

Martínez Nogueira, Roberto. (1999). Redes sociales. Más allá del individualismo y del comunitarismo. En Redes. El lenguaje de los vínculos (pp. 24-43). Buenos Aires, Argentina: Paidós.

Moscoloni, Nora. (2005). Las Nubes de Datos. Métodos para analizar la complejidad. Rosario, Argentina: UNR Editora.

Oficina Regional de Educación para América Latina y el Caribe- UNESCO. (2002). Revista PRELAC, áreas temáticas de trabajo: educación inclusiva. Recuperado el 10 de octubre de 2008 de http://www.unesco.cl/esp/atematica/educinclusiva/index.act

Perona Nélida, Rocchi Graciela. (2001). Vulnerabilidad y exclusión social. Una propuesta metodológica para el estudio de las condiciones de vida de los hogares. Recuperado en mayo 2007 de http://www.fices.unsl.edu.ar/ kairos/k08-08.htm.

Rovere, Mario. (2002). Hacia la conformación de redes en Redes. Hacia la construcción de redes en salud: los grupos humanos, las instituciones, la comunidad. Rosario, Argentina: Instituto de Salud Juan Lazarte y Secretaría de Salud Pública, Municipalidad de Rosario.

Toranzos, Liliana. (2000). El problema de la calidad en el primer plano de la agenda educativa. En OEI, Cumbre Iberoamericana Programa de Evaluación de la Calidad de Educación, (pp.36-45, Vol. 1). Buenos Aires: Ministerio de Cultura y Educación de la República Argentina.

Torres Castro, Carlos. (2005). Jóvenes y violencia. Revista Iberoamericana de Educación (37), 84-94. 